\title{
Dielectric signature of a dead layer in ultrathin films of
}

\section{a non-polar polymer}

\author{
Simone Napolitano, Michael Wübbenhorst \\ Katholieke Universiteit Leuven \\ Laboratory for Acoustics and Thermal Physics \\ Department of Physics and Astronomy \\ Celestijnenlaan 200D, B-3001 Leuven, Belgium \\ simone.napolitano@fys.kuleuven.be
}

The changes in the values of the expansion coefficients, shown for the two series in fig 2 of the text, were analyzed via a simple 3-layers model, based on the assumption that the bulk layer is sandwiched between a "localized" dead layer, of thickness $\delta$ with $\alpha_{\delta} \equiv 0$, at the interface with the substrate, and "localized" liquid like layer, $\xi$, being on top of the film at the interface with air, $\alpha_{\xi} \equiv \alpha_{M}^{\infty}>\alpha_{G}^{\infty}$; the apex ${ }^{\infty}$ indicates the bulk value.

Following the scheme proposed, the linear expansion coefficients can be approximated as sum of the three different components, linearly weighted by their respective volume fraction. The linearity of the composition is ensured by assuming that the interfacial interactions maintain constant the contact area between the polymer and the substrate. Under these conditions, lateral expansion is not allowed. 
The different trends observed in the two series obtained in different annealing conditions can be rationalized in terms of the role of a component with a higher mobility, and thus higher expansion coefficient. Assuming the presence of a liquid layer of constant extension present also in the glassy state, and that above $\mathrm{T}_{\mathrm{g}}$ the film can be schemed as a liquid layer on top of a dead layer, we can model the expansion coefficients as follow

$$
\begin{cases}T<T_{g} & \alpha_{G}(h)=\left(1-\frac{\delta+\xi}{h}\right) \cdot \alpha_{G}^{\infty}+\frac{\xi}{h} \cdot \alpha_{M}^{\infty} \\ T>T_{g} & \alpha_{M}(h)=\left(1-\frac{\delta}{h}\right) \cdot \alpha_{M}^{\infty}\end{cases}
$$

Under these conditions, upon reduction of the thickness, $\alpha_{G}$ increases, as $\alpha_{M}^{\infty}>\alpha_{G}^{\infty}$, while $\alpha_{M}$ shows a reduction. In this case the two expansion coefficients converge to the same values in correspondence of a critical thickness $h_{C} \equiv \delta+\xi$. Obviously, such a condition corresponds only to a fit extrapolation and cannot be reached experimentally as it is related to a null contrast, $\alpha_{M}\left(h_{C}\right)-\alpha_{G}\left(h_{C}\right)=0$

For films of series B, a 3-layers analysis yielded to $\delta=(2.7 \pm 0.5) \mathrm{nm}$ and $\xi=(1.6 \pm 0.5) \mathrm{nm}$; interestingly a previous determination of the thickness corresponding to the quantity $\alpha_{M} / \alpha_{G}=1$ for similar films gave a value of $5 \mathrm{~nm}$. ${ }^{\text {T }}$ This thickness corresponds to the quantity $\alpha_{M}-\alpha_{G}=0$ and thus to $h_{\mathrm{C}}$, which in our case is $(4.3 \pm 0.8) \mathrm{nm}$

Films of series A revealed a completely different trend. Both the expansion coefficients in the glassy state and the one in the melt decreased upon film thickness; moreover within the experimental errors, the same slope was found for the thickness dependence of reduced expansion coefficients both above and below $\mathrm{T}_{\mathrm{g}}$. The behavior can be rationalized by assuming that the annealing conditions used during

\footnotetext{
${ }^{a}$ Sharp JS, Teichroeb JH, Forrest JA EUROPEAN PHYSICAL JOURNAL E 15 (4): 473-487 DEC 2004
} 
the preparation of series A removed the components related to the liquid-like expansion coefficients $(\xi=0)$ and that a dead layer is formed on both interfaces.

In this case, the equations reported above become

$$
\begin{cases}T<T_{g} & \alpha_{G}(h)=\left(1-\frac{2 \delta}{h}\right) \cdot \alpha_{G}^{\infty} \\ T>T_{g} & \alpha_{M}(h)=\left(1-\frac{2 \delta}{h}\right) \cdot \alpha_{M}^{\infty}\end{cases}
$$

corresponding to the condition $\frac{\alpha_{G}(h)}{\alpha_{G}^{\infty}}=\frac{\alpha_{M}(h)}{\alpha_{M}^{\infty}}=\left(1-\frac{2 \delta}{h}\right)$ observed in the experimental data and reported in the text. The values obtained in the fit are comparable wit those previously reported in the literature.

A final remark on the fitting procedure is given in view of these considerations made in the text about the presence of a transition zone at the interface between bulk and dead layer.

The value found for the dead layer $\delta$ should be intended as a sum of two components, $\delta^{\prime}$ and $\lambda$, the former related to an ideal immobilized dead layer, the latter connected to the transition zone in which the molecular motions gradually recover the bulk behavior. The equations 1.2 can be thus generalized as

$$
\alpha_{i}=\left(1-2 \frac{\delta^{\prime}+\langle\lambda\rangle^{i}}{h}\right) \cdot \alpha_{i}^{\infty}+2\langle\lambda\rangle^{i} \alpha_{i}^{\lambda} \simeq\left(1-2 \frac{\delta^{\prime}+\frac{\langle\lambda\rangle^{i}}{2}}{h}\right) \cdot \alpha_{i}^{\infty}
$$

where the $\langle\lambda\rangle$ indicate the average value of the $\lambda$ in the temperature interval considered in the fit, and the index $i$ relates to the components in the glassy state $(\mathrm{G})$ and the melt $(\mathrm{M})$. The second equality is possible assuming that the layer $\lambda$ shows intermediate properties between those of $\delta$ ' and the ones of the bulk. 